\title{
The Globular Cluster System of NGC 5128
}

\section{Doug Geisler, ${ }^{1}$ Matias Gómez, ${ }^{1}$ W.E. Harris, ${ }^{2}$ K. Woodley, ${ }^{2}$ G. L. Harris, ${ }^{3}$ T. Puzia ${ }^{4}$ and M. Hempel ${ }^{5}$}

${ }^{1}$ Departamento de Fisica, Universidad de Concepción, Concepción, Chile email: dgeisler@astro-udec.cl

${ }^{2}$ Department of Physics and Astronomy, McMaster University, Hamilton ON L8S 4M1, Canada

${ }^{3}$ Department of Physics, University of Waterloo, Waterloo, Ontario, N2L 3G1, Canada ${ }^{4}$ Dominion Astrophysical Observatory, 5071 W. Saanich Rd, Victoria, BC V8X 4M6, Canada ${ }^{5}$ Department of Astronomy, University of Florida, Gainesville, FL 32611, USA

Abstract. We present preliminary results from a new wide-field photometric investigation of the globular cluster system of NGC 5128. NGC 5128 is the nearest giant elliptical and thus its globular cluster system serves as prototype for those of this important galaxy type. We have obtained images from IMACS at the Magellan telescope in excellent seeing that allow us to not only resolve globular clusters but derive their structural parameters. This extensive database provides a wealth of data to study the globular clusters in unprecedented detail. We discuss here the resolution of the clusters and their preliminary structural parameters.

Keywords. galaxies: star clusters, galaxies: individual (NGC 5128)

\section{Introduction}

Globular clusters (GCs) are ideal tracers to study galaxy formation. All massive galaxies have GCs. Giant ellipticals in particular can have thousands of them, and they are the brightest objects in these galaxies. They are ancient - many having formed before of during the time of the assembly of their host galaxy itself - but they can still form today in galaxies where the gas density and pressure is high enough. They are the supreme Simple Stellar Populations - with a unique age and metallicity - and as such are the quintessential tracers of the building blocks that are the subject of this conference. Indeed, they may well be these very building blocks! The derivation of key parameters like age and metallicity are well understood and calibrated. They are also extremely useful as dynamical tracers, especially of the dark matter content in the distant halo where no other tracers are available.

As the nearest (easily studied) giant elliptical galaxy, NGC 5128 (Centaurus A) possesses a GC system (GCS) that is a particularly important target for detailed studies of kinematics, abundances, ages, and other properties critical for investigating galaxy formation. It has the largest GCS of any galaxy within $15 \mathrm{Mpc}$. At a distance of only $3.8 \mathrm{Mpc}$ (Rejkuba 2004), its GCs are very bright $(V \sim 18-24)$. However, in spite of its great importance, the NGC 5128 GCS is still rather poorly studied. Indeed, we still do not even know its total GC population to better than $50 \%$ accuracy (Harris et al. 2004a,b, 2006). One of the reasons for this is in fact its proximity. Being so close, the GCS is spread out over $>1^{\circ}$, requiring very wide-field imagers to cover its entirety. An additional problematic factor is that it is at relatively low galactic latitude $\left(19^{\circ}\right)$ and relatively high galactic longitude $\left(310^{\circ}\right)$. These factors have prevented a truly global, detailed study of the NGC 5128 GCS until now. 
We recently attempted such a study using the CTIO $4 \mathrm{~m}$ Blanco telescope and the BTC camera (the precursor of the MOSAIC camera), which covers a similar $\sim 1 / 2^{\circ}$ field (Harris et al. 2004a,b). We imaged a very wide area, with complete coverage to $>1.2^{\circ}$, obtaining photometry for $>10^{5}$ objects in typical $>1$ " seeing. However, despite our use of the Washington photometric system, which provides a relatively clean separation of GCs from galaxies (Dirsch et al. 2003), we were unable to distinguish many stars from GCs, and were left with some $5 \times 10^{4}$ GC candidates, of which only $\sim 10^{3}$ are true GCs.

We realized that the best way to improve on this study and help eradicate as much of the huge contamination problem as possible was to supplement it with imaging from a camera that covers not only a wide-field but at very high spatial resolution and in very good seeing. This is because, since NGC 5128 is so close, a typical GC of several pc in physical size extends $\sim 0.5$ " in angular size and thus can be resolved in good seeing. We can then discriminate NGC 5128 GCs from stars via their resolution and also weed out a large additional fraction of galaxies by their physical appearance. Such a camera is provided by the IMACS instrument at Magellan.

\section{Observations and Reductions}

After several unsuccessful attempts, we finally obtained the requisite data. Images of NGC 5128 were obtained in April 2006 on the IMACS instrument at the Magellan Baade $6.5 \mathrm{~m}$ telescope at Las Campanas Observatory in Chile. The night was of excellent photometric quality and the seeing was superb. We obtained images in the $\mathrm{B}$ and $\mathrm{R}$ filters for a grid of $5 \times 5$ fields centered on the galaxy, with each field covering an area of 15.4 '. The pixel scale is 0.11 " and the seeing was $0.45 "$.

We followed the precepts established in Gómez et al. (2006) for reducing and analysing the data. Subtraction of a stellar psf from the initial image causes unresolved stars to disappear while resolved galaxies and GCs are left behind, the latter as "donuts", with oversubtracted centers and undersubtracted wings, which are easy to spot. Via this analysis, we confirmed the resolved nature of many previously-suspected GC candidates, especially from the BTC images. Additionally, we have uncovered a large number $\left(>10^{3}\right)$ new high quality GC candidates in NGC 5128. We have matched coordinates with the BTC data and thus combined the datasets to have BR and Washington $C T_{1}$ photometry for all candidates.

But our excellent seeing data allow us to do much more than this. In particular, we can derive structural parameters for GCs beyond the Local Group from groundbased images, as we did for the first time in Gómez et al. (2006) using similar but much smaller FOV data on the NGC 5128 GCS based on observations with the Magellan Magic camera, with a FOV of only 2'. We utilize the ISHAPE package written by S. Larsen, which convolves a King profile with the frame's psf to produce robust determinations of the GC's core radius, ellipticity, position angle and concentration. We derived structural parameters for all of the known and our new suspected GCs in NGC 5128.

\section{Preliminary Results}

Here we give some preliminary results from our analysis of the structural parameters of the innermost $3 \times 3$ IMACS fields, which have yielded 1133 GC candidates, based on their resolution and Washington $T_{1}, C-T_{1}$ indices. van den Bergh (2007) has recently compared the properties of 115 NGC 5128 GCs observed with HST by Harris et al. (2006) with those of the Galaxy and found suggestions of several important effects. First, he finds the most metal-poor NGC $5128 \mathrm{GCs}$, with $[\mathrm{Fe} / \mathrm{H}]<-1.8$, are on average 1.2 mags 


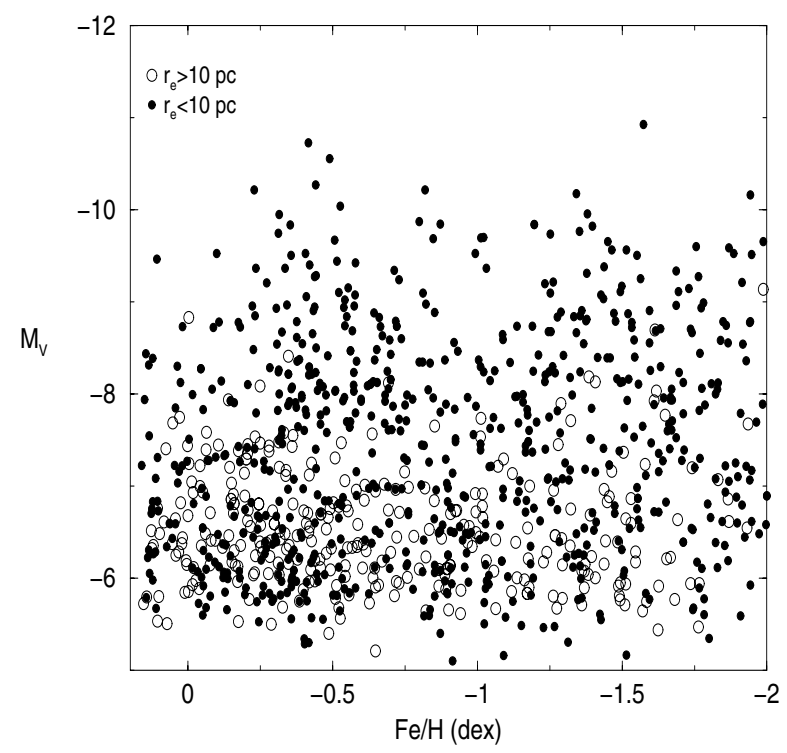

Figure 1. Metallicity (from the $C-T_{1}$ color) vs. $M_{V}$ for our GC candidates in NGC 5128. No trend is seen. However, smaller clusters are brighter than their larger cousins.

fainter than their more metal-rich cousins. No difference is seen in the Galactic GCs. Secondly, he finds that the larger clusters in both galaxies, with half-light radii $r_{h}>10$ pc, are fainter than smaller clusters. We have investigated these two effects with our new dataset, which does not have the resolution of HST but is a factor 10 larger in sample size. Figure 1 shows that 160 GCs with $\left[\mathrm{Fe} / \mathrm{H}<-1.8\right.$ have the same $M_{V}$ as 973 more metal-rich GCs. It also shows that the larger clusters are indeed fainter than their smaller counterparts. Thus, we find the NGC 5128 GCS to be more like the Galactic GCs than suggested by van den Bergh.

A growing body of evidence finds that metal-rich GCs in extragalactic systems are typically $\sim 20 \%$ smaller than metal-poor clusters (e.g. Kundu et al. 1999, Jordan 2004). However, in our initial study of the structural parameters for NGC 5128 GCs from the much smaller Magic database (Gómez et al. 2006), we found a significant hint that the metal-rich GCs were actually larger than the metal-poor GCs. Our preliminary analysis for our new IMACS sample bears this out, as shown in Figure 2. Some corroboration of this effect comes from the recent work of Spitler et al. (2006) on the GCS of the Sombrero galaxy. This study suggests that the size difference may only hold in the inner few kpc of the galaxy and that beyond this the GCs may be similar in size. The reported difference may be due in part to projection effects, as first suggested by Larsen \& Brodie (2003). Determining the nature and origin of any differences in size between different GC subpopulations is of great importance, both in our theoretical understanding of their formation and evolution as well as for the suggested use of sizes as a distant indicator (Jordan 2004).

We are following up this extensive database of new GC candidates in NGC5128 by obtaining optical spectroscopy. We have already observed a large number of them with the CTIO 4m + Hydra, NTT + EMMI, Magellan + LDSS-2 and Gemini-S + GMOS. In addition, we will soon obtain more data with Hydra, GMOS, VLT + VIMOS and Magellan + IMACS. The aim is to derive radial velocities for at least $1 / 2$ of the GCS in order to use them to explore the structure of the dark matter halo at large radii, compare 


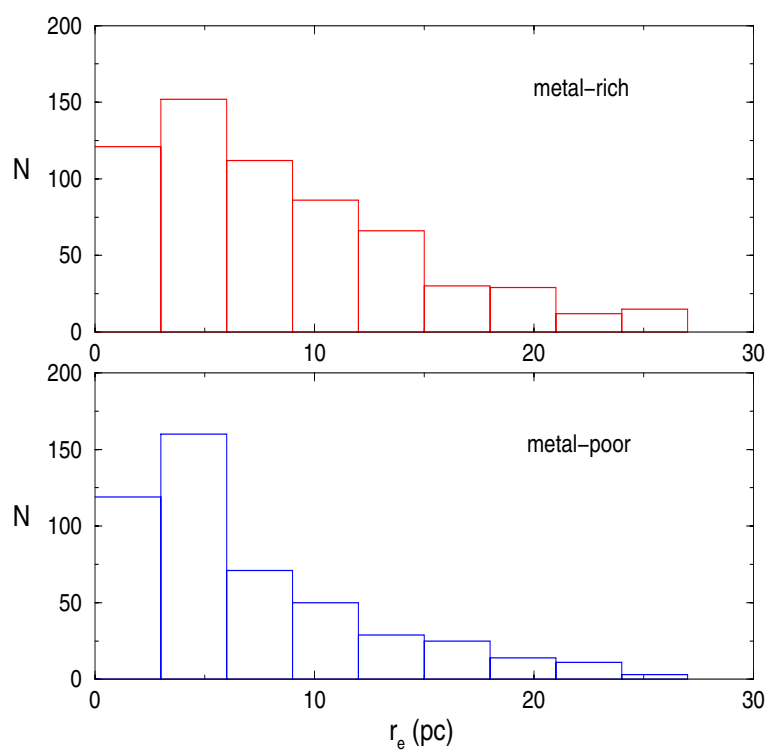

Figure 2. Histograms of the sizes of our new IMACS GC sample for both metal-rich (top) and metal-poor (bottom) subpopulations. The metal-rich GCs are significantly larger in the mean.

the kinematics of the metal-rich and -poor subpopulations, etc. We will also obtain much higher $\mathrm{S} / \mathrm{N}$ spectra for a bright subset of the GCs to determine their detailed ages, metal abundances, $[\alpha / F e]$ and other chemical ratios. This should enable us to derive the definitive study of the kinematics, dynamics, age and abundance distributions for the NGC 5128 GCS and provide very strong constraints on galaxy formation theories.

\section{Acknowledgements}

D.G. gratefully acknowledges support from the Chilean Centro de Astrofísica FONDAP No. 15010003.

\section{References}

Dirsch, B., Richtler, T., Geisler, D., et al., 2003, AJ 125, 1908

Gómez, M., Geisler, D.; Harris, W. E.; Richtler, T.; Harris, G. L. H.; \& Woodley, K. A. 2006, $A \mathscr{G} A 447,877$

Harris, G., Geisler, D., \& Harris, W.E. 2004a, AJ 128, 712

Harris, G., Harris, W.E., Geisler, D., et al. 2004b, AJ 128, 723

Harris, W.E., Harris, G.L.H., Barmby, P., McLaughlin, D., \& Forbes, D.A. 2006 AJ 132, 2187 Jordan, A. 2004, ApJ 613L, 117

Kundu, A., Whitmore, B.C., Sparks, W.B., Macchetto, F.D., Zepf, S.E., \& Ashman, K.M. 1999, ApJ 513, 733

Larsen, S.S., \& Brodie, J.P. 2003, ApJ 593, 340

Rejkuba, M. 2004, A\&A, 413, 903

Spitler, L.R., Larsen, S.S., Strader, J., Brodie, J.P., Forbes, D.A., \& Beasley, M.A. 2006, AJ 132,1593

van den Bergh, S. 2007, $A J$ in press

\section{Discussion}

SeтH: The relations fo size and luminosity and metallicity are both what would be expected for background galaxies, have any of your spectra confirmed this trend?. 
GEISLER: Too early to say yet but our studies suggest that contamination by galaxies should only be at the $\sim 10$ per cent level.

Peterson: Can you get velocity dispersions from your spectra?. Especially good since you will have structural parameters you could constrain M/L ratios.

GEISLER: No, our spectra are not high enough resolution. A few such spectra and results have been published by Ho et al. We intend to propose to get such spectra for some of the brightest clusters.

MCDERMID: Is there evidence from the globular clusters observed that NGC5128 has experienced a recent interaction?.

GEISLER: Yes, there are few very blue clusters observed that may have been created in the merger.

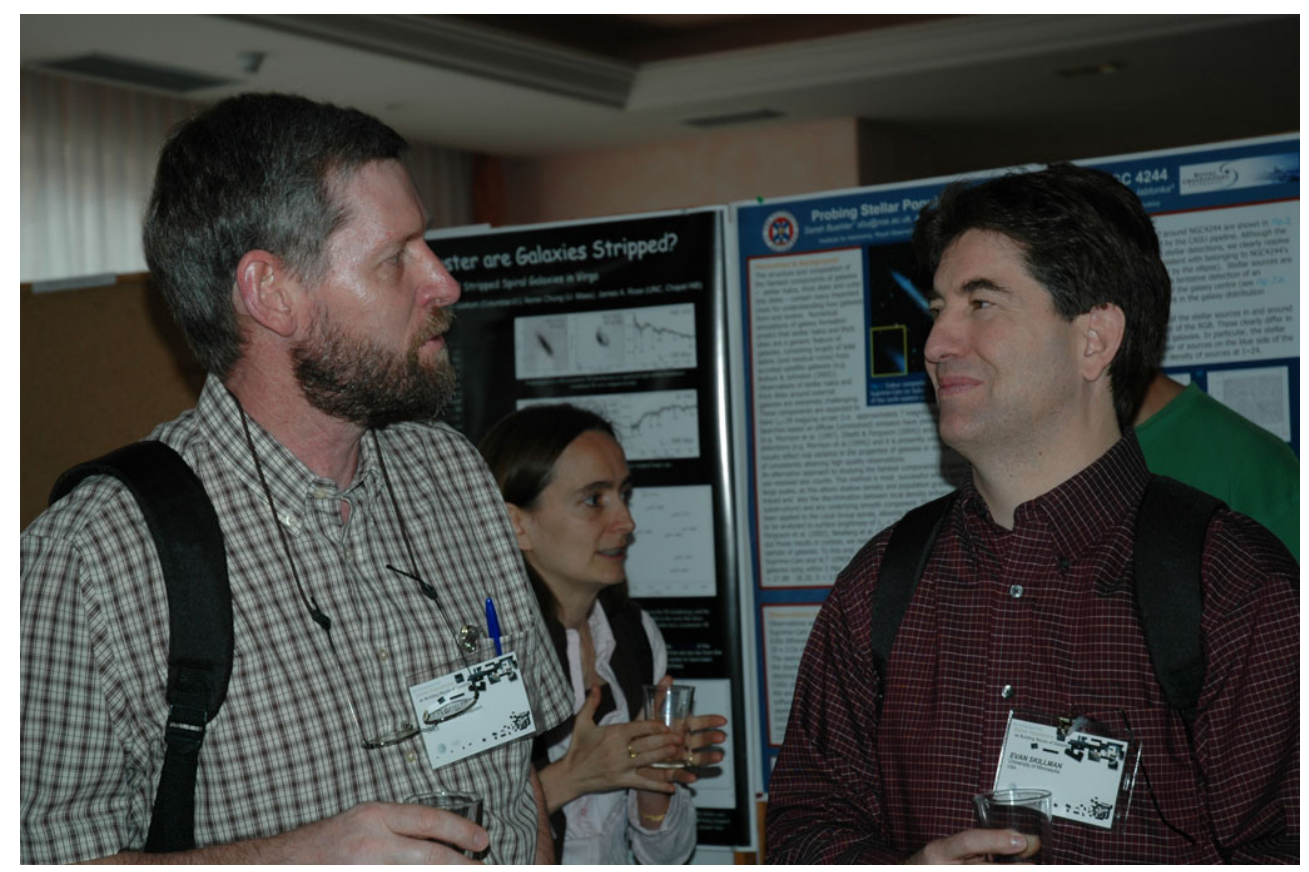

The speaker (left) with Evan Skillman. In the back: Arian Lançon. 